\title{
Analysis of sandwich beam structures using kriging based higher order models
}

\author{
M.A.R. Loja ${ }^{\mathrm{a}, \mathrm{b}, *}$, J.I. Barbosa ${ }^{\mathrm{a}, \mathrm{b}}$, C.M. Mota Soares ${ }^{\mathrm{b}}$ \\ a ISEL, IPL, Av. Conselheiro Emídio Navarro, 1, 1959-007 Lisboa, Portugal \\ ${ }^{\mathrm{b}}$ LAETA, IDMEC, Instituto Superior Técnico, Universidade de Lisboa, Av. Rovisco Pais, 1, 1049-01 Lisboa, Portugal
}

\section{A R T I C L E I N F O}

\section{Article history:}

Available online 20 August 2014

\section{Keywords:}

Kriging interpolation

Higher-order shear deformation theory

Structural analysis

Bossak-Newmark method

Functionally graded sandwich structures

Mori-Tanaka homogenization

\begin{abstract}
A B S T R A C T
Functionally graded composite materials can provide continuously varying properties, which distribution can vary according to a specific location within the composite. More frequently, functionally graded materials consider a through thickness variation law, which can be more or less smoother, possessing however an important characteristic which is the continuous properties variation profiles, which eliminate the abrupt stresses discontinuities found on laminated composites. This study aims to analyze the transient dynamic behavior of sandwich structures, having a metallic core and functionally graded outer layers. To this purpose, the properties of the particulate composite metal-ceramic outer layers, are estimated using Mori-Tanaka scheme and the dynamic analyses considers first order and higher order shear deformation theories implemented though kriging finite element method. The transient dynamic response of these structures is carried out through Bossak-Newmark method. The illustrative cases presented in this work, consider the influence of the shape functions interpolation domain, the properties through-thickness distribution, the influence of considering different materials, aspect ratios and boundary conditions.
\end{abstract}

() 2014 Elsevier Ltd. All rights reserved.

\section{Introduction}

In many different areas of knowledge, it is of fundamental importance not only to obtain an adequate geometric and spatial representation of the object or structure under study but also to be able to proceed in a continuous processing pipeline to subsequent analyses of its structural performance behavior. Concerning to the first stage devoted to the geometrical modeling, it is common to encounter this need closely related to field acquisition of data, namely by using laser scanning or other optical techniques, in areas that range from the meteorology, mining, water and other natural resources to architecture and heritage conservation and rehabilitation, structural or biomedical engineering. From the point clouds acquired through the mentioned techniques it is possible to extract information about the coordinates of each point as well as the colours associated and in some cases that mainly depend on the equipment used also other additional characteristics. Concerning to this stage, different researchers have presented aiming to establish methodologies to a representative modeling of

\footnotetext{
* Corresponding author at: ISEL, IPL, Av. Conselheiro Emídio Navarro, 1, 1959-007 Lisboa, Portugal.

E-mail addresses: amelialoja@dem.isel.ipl.pt, amelialoja@tecnico.ulisboa.pt (M.A.R. Loja).
}

different types of objects or structures. One of these works is due to Karbowski [1] which presented a reverse engineering system that uses geostatistical kriging interpolation method, to process the points cloud acquired by a coordinate measuring system. The system developed enables the comparison of two point's clouds, allowing the modification of machining programs for a 3-axis milling centre and the improvement of the reproducing workpiece. Also related to the nature of the approximating process and the nature of the surface it is worth mentioning the study carried out by Martin and Simpson [2]. These authors analyzed different forms of kriging computational models to understand if more complex forms are more accurate and easier to fit than simple forms of kriging models. To this purpose they proceeded to comparisons between the maximum likelihood estimation, and cross-validation parameter estimation in order to select kriging model's parameters. Due to its characteristics, kriging method has also been used to construct metamodels or surrogate models in various scientific areas. To illustrate this fact in the structures reliability field, we can refer the work developed by Kaymaz [3]. In his paper it is investigated the use of the kriging method for structural reliability problems by comparing it with the response surface method based on least square regression. Kriging parameters are also examined on the basis of the $\hat{a}$ computation and fitting behavior. The authors 
additionally suggest some points for which the kriging model may be improved to get better results for structural reliability problems. As in other contexts and subjects, the appropriateness of kriging and of any other method based on probabilistic models to represent spatial data is frequently questioned. A nonstochastic approach that sometimes is presented as an alternative is the use of spline functions. Laslett [4] studied the precision of the predictions of these approaches, based on real data. To this effect he divided data sets into modeling sets and prediction sets. According to the author, in the cases examined, kriging can outperform splines by a considerable margin, and it never performs worse than splines. Splines have also been used to predict or reconstruct surfaces and solids, as well as to predict the behavior of structures. One example of this later usage is due to Loja et al. [5] where optimal configurations of structures modeled with B-splines, were further obtained through the use of genetic algorithms optimization technique. A more recent work focused on the coupled static and dynamic buckling behavior of thin walled beams using spline functions was presented by Yang et al. [6].

Focusing in the next step of the mentioned pipeline going from geometrical modeling to structural analyses and characterization of performance behavior, it remains a significant work to do. In fact in some scientific areas, these stages are developed usually by different applications with limited or inexistent interconnectivities. This is partially explained by the great volume of data in the reconstruction of the surfaces and thus on the specialization of the applications, but also because interdisciplinary approaches need to be improved. Maintaining our focus on kriging method because of its potential concerning this link, it is possible to find the first use of kriging functions to enhance the finite element method in the prediction of the structural behavior, by Plengkhom and KanokNukulchai [7]. On their paper the finite element method adopted the moving kriging interpolation as a substitute for the traditional functions, proposing a concept of layered domain of influence. The possibility to introduce this concept on existing finite element methods was empathized. Following this approach, Bui and Nguyen [8] used a meshfree method for free vibration analysis of classical Kirchhoff's plates. The deflection of plates is approximated by the moving Kriging interpolation method. As these functions possess Kronecker's delta property, the method reveals to be efficient and straightforward in imposing the essential boundary conditions, and no special treatment techniques are required. In another work, Bui et al. [9] considered the same approach to study the free and forced vibrations of two-dimensional solids. The governing elastodynamic equations were transformed into a standard weak formulation, and then discretized into a meshfree system of timedependent equations, which are solved by the standard implicit Newmark time integration scheme. Because of its importance, other authors have studied the dynamic responses of different types of structures. Focusing on the broad field of structures made of composite materials, we can refer among others, the work developed by Reddy [10] which studied the transient response of isotropic, orthotropic and layered anisotropic composite plates modeled with shear-flexible finite elements. Numerical convergence and stability of the element was established using Newmark's direct integration technique. The parametric effects of the time step, finite element mesh, lamination scheme and orthotropy on the transient response were investigated. Also, within this subject Kant et al. [11] and Nayak et al. [12] presented important works on the characterization of the dynamic answer of composite laminated structures. More recently, Neves et al. [13] carried out a study upon functionally graded shells by using a meshless approach. In their work the analysis is performed by radial basis functions collocation, according to a higher-order shear deformation theory that accounts for throughthickness deformation. The equations of motion and the boundary conditions are obtained by Carrera's Unified Formulation resting upon the principle of virtual work, and further interpolated by collocation with radial basis functions. Also based on the use of a meshfree approach, resulting from the combination of pseudospectral methods and a collocation method, using radial basis functions, Roque et al. [14] presented a work dealing with the solution of time-dependent problems. In that work the transient answer of functionally graded plates and shells was analyzed by using Newmark method [15]. On the present work the authors consider the study of the dynamic behavior of a beam-type structure with a sandwich constitution. The average material properties of the sandwich which outer layers are made of functionally graded materials are estimated through Mori-Tanaka homogenization scheme [16]. The static and dynamic analyses are carried out using kriging finite element models that were developed based on Lo et al. [18] displacement field and on the first order shear deformation field. The dynamic behavior characterization is carried out by using the method presented in Wood et al. [19] known as Bossak-Newmark method in the literature. The performance of the models is illustrated and characterized through a set of case studies.

\section{Kriging based finite element models}

Kriging is a form of generalized regression for the formulation of an optimal estimator, in a similar sense to that corresponding to the least squares method. This geostatistical technique was first used for spatial interpolation in mining and geology, by Krige [20] and it became also an important tool in the more generic field of surfaces modeling in reverse engineering. The method is based on the assumption that to closer locations correspond similar data results, and considering that any unknown node value can be then interpolated by using neighbor scattered points. In the present work, the geometrical characteristics as well as the behavior of the beam-type structure are modeled using kriging. The finite element models considered on the present study use shape functions which were derived from the kriging interpolation technique.

These interpolation functions are adequate to be used within finite element method as they have the Kronecker delta and consistency properties $[7,8]$ and the adaptation of an existing finite element code, to be able to use these functions is easily carried out. In Fig. 1 we illustrate some shape functions associated to the interpolation domain $[-1, \ldots, 1]$, using quadratic and cubic polynomial basis respectively. The curves depicted are a sub-set of the functions derived and used on this work. As can be observed in Fig. 1, the functions whose curves are depicted, refer to equally spaced nodes, however this is not compulsory. Assuming that a generic function $q(x)$, for instance a degree of freedom, can be approximated by a linear combination of functions $\phi$ and the values assumed by each function in the nodal points pertaining to the interpolation domain, we can write:

$$
\begin{aligned}
& \bar{q}(x)=\phi(x) \cdot q(x) \\
& \phi(x)=p^{T}(x) \cdot A+r^{T}(x) \cdot B
\end{aligned}
$$

where $x$ denotes the nodal values vector, associated to a subdomain considered within a scattered points domain, and matrices $A$ and $B$ are given as:

$$
\begin{aligned}
& A=\left(P^{T} \cdot R^{-1} \cdot P\right)^{-1} \cdot P^{T} \cdot R^{-1} \\
& B=R^{-1}(I-P \cdot A)
\end{aligned}
$$

Matrices $P$ and $R$ are defined as follows:

$$
P=\left[\begin{array}{ccc}
p_{1}\left(x_{1}\right) & \cdots & p_{M}\left(x_{1}\right) \\
\cdots & \cdots & \cdots \\
p_{1}\left(x_{N}\right) & \cdots & p_{M}\left(x_{N}\right)
\end{array}\right], \quad R=\left[\begin{array}{ccc}
\mathcal{R}\left(x_{1}, x_{1}\right) & \ldots & \mathcal{R}\left(x_{1}, x_{N}\right) \\
\ldots & \cdots & \cdots \\
\mathcal{R}\left(x_{N}, x_{1}\right) & \cdots & \mathcal{R}\left(x_{N}, x_{N}\right)
\end{array}\right]
$$



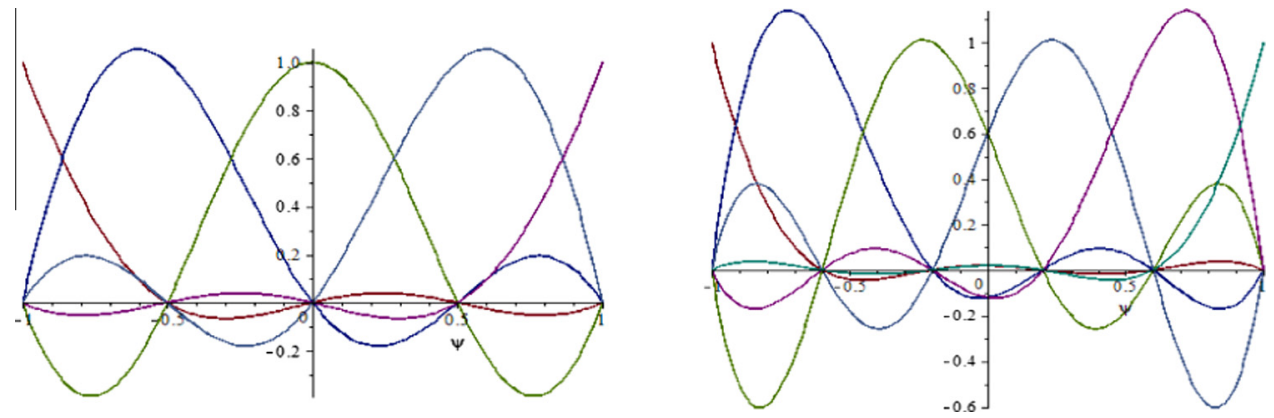

Fig. 1. Examples of kriging shape functions.

In general, matrix $P$ is a rectangular matrix where $M$ represents the order of the polynomial basis considered on the study. For instances if we have the one-dimensional first order polynomial basis $p^{T}(x)=\left[\begin{array}{lll}1 & x & x^{2}\end{array}\right]$, then we will have $M=2$, and for the case of the second order basis $p^{T}(x)=\left[\begin{array}{llll}1 & x & x^{2} & x^{3}\end{array}\right]$, then we have $M=3$. Concerning to the parameter $N$, it represents the number of points in the domain used for the establishment of the shape function $\phi(x)$. In the present study, the covariance coefficients $R\left(x_{i}, x_{j}\right)$ of the correlation matrix $R$, were determined using the Gaussian function, $\exp \left(-\theta \cdot r_{i j}^{2}\right)$, where $r_{i j}$ is the Euclidean distance between two given points.

\section{Shear deformation theories}

Considering the interest on studying more general situations where the classical theory restrictions are not adequate to be adopted, the present work is based on Lo et al. [18] displacement field (HSDT). So, for the type of structures which are going to be modeled in this study, the displacement field assumes the following expression:

$$
\begin{aligned}
& u(x, z, t)=u^{0}(x, t)+z \cdot \theta_{x}^{0}(x, t)+z^{2} \cdot u^{0 *}(x, t)+z^{3} \cdot \theta_{x}^{0 *}(x, t) \\
& w(x, z, t)=w^{0}(x, t)+z \cdot \beta^{0}(x, t)+z^{2} \cdot w^{0 *}(x, t)
\end{aligned}
$$

where the degrees of freedom $u^{0}, w^{0}, \theta_{x}^{0}$ denote the displacements along the $x$ and $z$ directions and the rotation around the $y$ axis, while the $u^{0 *}, w^{0 *}, \beta^{0}, \theta_{x}^{0 *}$ represent higher order modes of deformation that result from the Taylor series expansion of the displacement field. If one proceeds to the elimination of these higher order terms we obtain the first order shear deformation theory (FSDT) displacement field that was also implemented in the present work. In the case of the FSDT model, unlike the HSDT, it is required the use of a shear correction factor because of its inherent constant distribution shear strain profile. Each of the models can use a different number of interpolation functions, for instance the FSDT model can use a three nodes or a five nodes domain, considering either linear or quadratic polynomial basis. For the HSDT model, which can consider either quadratic or cubic polynomial basis, one can vary to the three or four nodes domain to a five or six nodes interpolation domain

\section{Functionally graded material properties}

For a generic sandwich beam with a metallic core and outer faces made of a functionally graded material, where the properties are assumed to depend on the thickness coordinate $z$ following an exponent power law, the distribution of metal volume fraction can be written as:

$V_{m}(z)= \begin{cases}\left(\frac{z-z_{\text {inf }}}{z_{1}-z_{\text {inf }}}\right)^{p \text { inf }}, & z \in\left[z_{\text {inf }}, z_{1}\right] \\ 1, & z \in\left[z_{1}, z_{2}\right] \\ \left(\frac{z-z_{\text {sup }}}{z_{2}-z_{\text {sup }}}\right)^{p \text { sup }}, & z \in\left[z_{2}, z_{\text {sup }}\right]\end{cases}$ where $z_{\text {inf }}$ and $z_{\text {sup }} h_{\text {inf }}$ are the coordinates of the lower and upper outer surfaces of the sandwich and $z_{1}$ and $z_{2}$ are respectively the coordinates associated to the interfaces between the inferior layer and the core, and between the core and the superior layer, as can be observed in Fig. 2.The effective composite properties are calculated using Mori-Tanaka estimate [16], and considering the previously referred metal volume fraction distribution. This approach approximates the interaction between phases, assuming the stress in each phase equals the stress in a singular inclusion within the embedding matrix, submitted to a mean strain field applied to that matrix. The effective properties can thus be obtained by solving the equations:

$E_{e f f}=\frac{9 K G}{3 K+G} ; \quad v_{e f f}=\frac{3 K-2 G}{2(3 K+G)} ; \quad G_{e f f}=\frac{E_{e f f}}{2\left(1+v_{\text {eff }}\right)}$

where the bulk modulus $(K)$ and shear modulus $(G)$ are obtained as:

$\frac{K-K_{m}}{K_{c}-K_{m}}=V_{c} /\left(1+V_{m}\left(\frac{K_{c}-K_{m}}{K_{m}+\frac{4}{3} G_{m}}\right)\right)$,
$\frac{G-G_{m}}{G_{c}-G_{m}}=V_{c} /\left(1+V_{m}\left(\frac{G_{c}-G_{m}}{G_{m}+\frac{G_{m}\left(9 K_{m}+8 G_{m}\right)}{6\left(K_{m}+2 G_{m}\right)}}\right)\right)$

$K_{m}=E_{m} / 3\left(1-2 v_{m}\right), \quad K_{c}=E_{c} / 3\left(1-2 v_{c}\right)$,

$G_{m}=E_{m} / 2\left(1+2 v_{m}\right), \quad G_{c}=E_{c} / 2\left(1+2 v_{c}\right)$

The effective specific mass distribution is determined by using the Voigt mixtures law, which is generically expressed as:

$P_{\text {eff }}=P_{m} V_{m}+P_{c} V_{c}, \quad V_{c}=1-V_{m}$

This rule of mixtures states that the effective material property $P_{\text {eff }}$, for instance the density, is associated to a generic thickness coordinate $z$ and depends on the corresponding metal and ceramic properties $P_{m}$ and $P_{c}$ weighted by the corresponding volume fractions $V_{m}$ and $V_{c}$.

\section{Transient dynamic analysis}

The equations of motion for a linear structural dynamic problem using the finite element method can be written as in Reddy [17]:

$M \ddot{u}(t)+C \dot{u}(t)+K u(t)=F(t)$

where $M, C$ and $K$ are respectively the mass, damping and stiffness matrices of the structure, and $F$ represents the vector of the applied

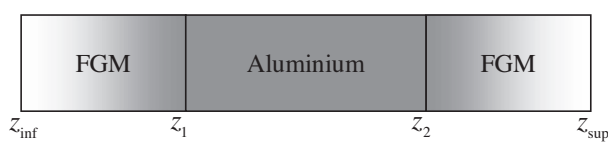

Fig. 2. Sandwich configuration through-thickness. 
loads. The displacement vector $u$ is a function of time, and its first and second time derivatives are represented by superposing dots notation on $u$.

The numerical method used in the present work to integrate the equations of motion is due to Bossak-Newmark method [19] which is an extension of Newmark method [15]. The main reason to use this method is related to the fact that it considers acceleration, velocity and displacement vectors at only two time levels, also providing a positive artificial damping of higher modes and a good phase following when compared to the very often used Newmark method.

This artificial damping of higher modes can be an important issue when the number of degrees of freedom and thus the natural frequencies, is high. However many of those modes, some of them related to the nature of the discretization assumed, can be neglected because they are not that representative of the real dynamic behavior of the structure. Thus it is considered to be adequate to introduce some artificial numerical damping to remove the participation of high frequency components. In Bossak-Newmark method, the equations of motion can be re-written as an equation system constituted as follows:

$\widehat{K}_{k+1} u_{k+1}=\widehat{F}_{k+1}$

$\widehat{K}_{k+1}=K+b_{1} M+b_{2} C$

$\widehat{F}_{k+1}=F_{k+1}+M\left(b_{1} u_{k}+b_{3} \dot{u}_{k}+b_{4} \ddot{u}_{k}\right)+C\left(b_{2} u_{k}+b_{5} \dot{u}_{k}+b_{6} \ddot{u}_{k}\right)$

where $\hat{K}_{k+1}$ and $\hat{F}_{k+1}$ denote respectively the effective stiffness and the effective load vector at instant $k+1$, and the $b_{i},(i=1, \ldots, 6)$ parameters are given as:

$b_{1}=\frac{1-\alpha_{B}}{\beta_{B} \Delta t^{2}}, \quad b_{2}=\frac{\gamma_{B}}{\beta_{B} \Delta t}, \quad b_{3}=\frac{1-\alpha_{B}}{\beta_{B} \Delta t}$,

$b_{4}=\frac{1}{2 \beta_{B}}-\frac{\alpha_{B}}{2 \beta_{B}}-1, \quad b_{5}=\frac{\gamma_{B}}{\beta_{B}}-1, \quad b_{6}=\frac{\gamma_{B}}{\beta_{B}}-2$

The solution of the system yields the vector $u_{k+1}$ and the corresponding velocities and accelerations are updated as:

$\ddot{u}_{k+1}=\frac{1}{\beta_{B} \Delta t^{2}}\left(u_{k+1}-u_{k}\right)-\frac{1}{\beta_{B} \Delta t} \dot{u}_{k}-\left(\frac{1}{2 \beta_{B}}-1\right) \ddot{u}_{k}$

$\dot{u}_{k+1}=\dot{u}_{k}+\Delta t\left(1-\gamma_{B}\right) \ddot{u}_{k}+\Delta t \gamma_{B} \ddot{u}_{k+1}$

Stability conditions are satisfied when $\alpha_{B} \leqslant 0.5, \beta_{B} \geqslant \gamma_{B} \geqslant 0.25$, $\alpha_{B}+\gamma_{B} \geqslant 0.25$. If we consider $\alpha_{B}=0$ then Bossak-Newmark method reduces to Newmark method with $\beta_{B}=\beta, \gamma_{B}=\gamma$ Newmark parameters. Both approaches were used on the present work.

\section{Numerical applications}

The studies here presented consider a sandwich beam rectangular transverse section, with an aluminum ( $\mathrm{Al})$ core and outer layers made of a ceramic-metal mixture reaching at the outer surfaces, a ceramic-rich silicon carbide $(\mathrm{SiC})$, zirconia $(\mathrm{ZrO} 2)$ or alumina (Al2O3) composition, according to Eq. (6) and observable in Fig. 2. The properties of these materials used in this study are presented in Table 1.

where $E, v, \rho$ stands for the Young's modulus, Poisson's ratio and density. The thickness of each inferior and superior layer is

Table 1

Material properties.

\begin{tabular}{lcll}
\hline Material & $E(\mathrm{GPa})$ & $v$ & $\rho\left(\mathrm{kg} / \mathrm{m}^{3}\right)$ \\
\hline Aluminum (Al) & 70 & 0.3 & 2702 \\
Zirconia (ZrO2) & 200 & 0.3 & 5700 \\
Silicon carbide (SiC) & 420 & 0.3 & 3100 \\
Alumina (Al2O3) & 380 & 0.3 & 3800 \\
\hline
\end{tabular}

equal to $0.01 \mathrm{~m}$ and the core thickness is equal to $0.02 \mathrm{~m}$, being considered different length to thickness ratios. The analyses carried out and illustrated in the present work, intend to characterize the influence on the structure dynamic response, of parameters such as: the shear deformation theory, the interpolating functions and its relation to the interpolation domain, the aspect ratio $L / h$ (length/total thickness), the material properties distributions as well as the usage of different materials, and also the numerical method used to carry out the numerical integration of the equations of motion in a dynamic context.

\subsection{Case 1: convergence characteristics of models}

The first case considers a sandwich strip which outer layers are constituted of a combination of aluminium and silicon carbide, according to the metal volume fraction distribution mentioned in Section 4.

In this case we use Voigt rule of mixtures to determine the sandwich properties. Concerning to the value of the exponent power law, it is used the case $p=6$, and a shear correction factor of 5/6. The strip has a length to thickness ratio of ten, it is clamped on both ends and it is submitted to a uniform distributed loading $q_{0}=10^{4} \mathrm{~N} / \mathrm{m}$. The convergence patterns obtained for the different models are depicted on Fig. 3, where the models are identified by a scheme sequence "XXXX YZ".

The first four characters "XXXX" represent the shear deformation theory used, the fifth " $Y$ " stands for the order of the polynomial basis ( $\mathrm{L}$ for linear, $\mathrm{Q}$ for quadratic, $\mathrm{C}$ for cubic) and the last character " $Z$ " indicates the number of shape functions in the interpolation domain. Considering the FSDT models, the L3 one is the model that presents a greater discrepancy for coarse meshes, a similar conclusion can be drawn related to the HSDT Q3. This situation can be minimized if maintaining however the polynomial basis order, we increase the number of the interpolating functions used within the domain (FSDT L5 and HSDT Q5 respectively) which is an expected trend. If however higher order polynomial basis are considered, it is also possible to see that moderately refined meshes can give satisfactory results. This is the case for instance of FSDT Q3 and Q5 and also of HSDT Q5, C4 and C6, neglecting the differences associated to the theory used.

For comparison purposes, the solution obtained for this case, using B-spline strip finite elements [5] is $3.182 \mathrm{E}-7 \mathrm{~m}$ and $3.290 \mathrm{E}-7 \mathrm{~m}$ respectively for the 4LFSDT6 and 4CFSDT6 models. In this simulation, ten sections were considered. As can be seen in more detail, in Loja et al. [5], both models, 4LFSDT6 and 4CFSDT6, are based on first order shear deformation theory and implemented through two nodal line's strips, where the first considers linear B-spline functions and the second model uses cubic $\mathrm{B}$-spline functions in the longitudinal direction.

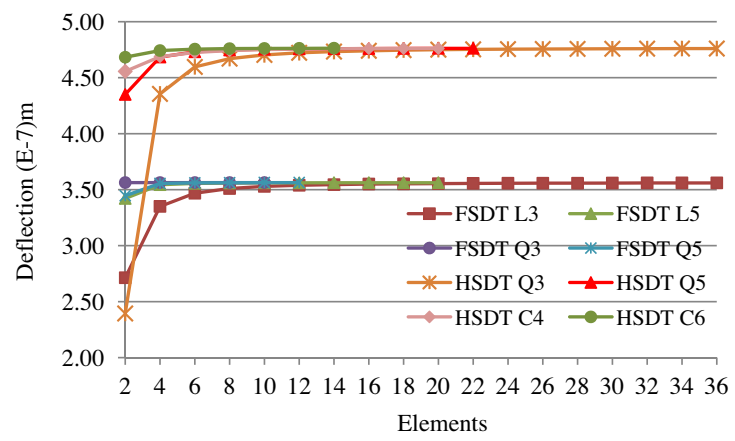

Fig. 3. Convergence of kriging finite element models implemented. 


\subsection{Case 2: deflection of clamped-clamped sandwich strip}

This second study considers the same sandwich strip, and compares the results obtained with the different models implemented in the present work with alternative ones. The maximum deflections predicted by the FSDT models, can be observed in Table 2 where they are also compared with an FSDT based analytical solution [21] for different values of length-thickness ratios and shear correction factors $(k)$. A discretization in twelve elements was used.

From this table it is possible to conclude that the results obtained with the FSDT kriging finite element models present a fair agreement with the analytical results although less conservative. Concerning to the present HSDT models, they present a more flexible behavior, as expected, and the maximum deflections obtained can be observed in Table 3 .

In this case, for comparison purposes, the 3D solutions obtained by Nguyen et al. [21] using five discrete layers are also included. The implemented HSDT models are equivalent single layer based, so the deviations obtained could be expected, with a particular significance to the thick beam.

\subsection{Case 3: dynamic response of clamped-clamped thin beam}

A thin isotropic beam $(L / h=100)$ clamped at both ends is now considered. This beam is subjected to an initial deflection, expressed as, $w(x, 0)=\sin (\pi x)-\pi x(1-x)$. The beam has a unit length and a rectangular cross section and it obeys to the following relations: $E I=1.0$ and $\rho A=1.0$ (where $E$ stands for Young's modulus, $I$ for the second moment of area, $\rho$ for the density and $A$ for the cross section area).

Accordingly we get $G A k=4 E I / h^{2}$ where $G$ is the shear modulus and $k$ the shear correction factor. In the present case it is considered $k=5 / 6$ and $v=0.25$. The transient response obtained by [17] using a first order shear deformation model and the transverse displacement distributions obtained through some of the present FSDT models, can be observed on Fig. 4.

From the curves depicted on this figure it is possible to conclude from a fair agreement among the present FSDT Q3 and Q5 models and Reddy solution, which considered symmetry and has used two quadratic beam elements, referenced as $4 \mathrm{Q}$.

\subsection{Case 4: influence of different ceramic materials and material distribution}

To characterize the influence of the functionally graded mixtures metal-ceramic in the outer layers as well as its distribution, a set of situations was analyzed using FSDT Q5 model. The aspect ratio of a clamped-clamped sandwich strip was to $10(L / h)$. The maximum deflections and the fundamental frequencies obtained can be observed in Table 4 .

The value of the power law exponent $(p)$ which rules the material distribution through the thickness shows to have an important influence on both the quantities analyzed. As this parameter increases, the maximum deflections decrease and the fundamental frequencies also increase. This is an expected behavior. Also we can
Table 3

Strip maximum deflections for HSDT models.

\begin{tabular}{rlllll}
\hline \multirow{2}{*}{$L / h$} & \multicolumn{5}{l}{ Maximum deflections (m) } \\
\cline { 2 - 6 } & FEM [21] & HSDT Q3 & HSDT Q5 & HSDT C4 & HSDT C6 \\
\hline 5 & $6.023 \mathrm{e}-8$ & $5.392 \mathrm{E}-8$ & $5.416 \mathrm{E}-8$ & $5.417 \mathrm{E}-8$ & $5.418 \mathrm{E}-8$ \\
10 & $4.822 \mathrm{e}-7$ & $4.703 \mathrm{E}-7$ & $4.754 \mathrm{E}-7$ & $4.751 \mathrm{E}-7$ & $4.761 \mathrm{E}-7$
\end{tabular}

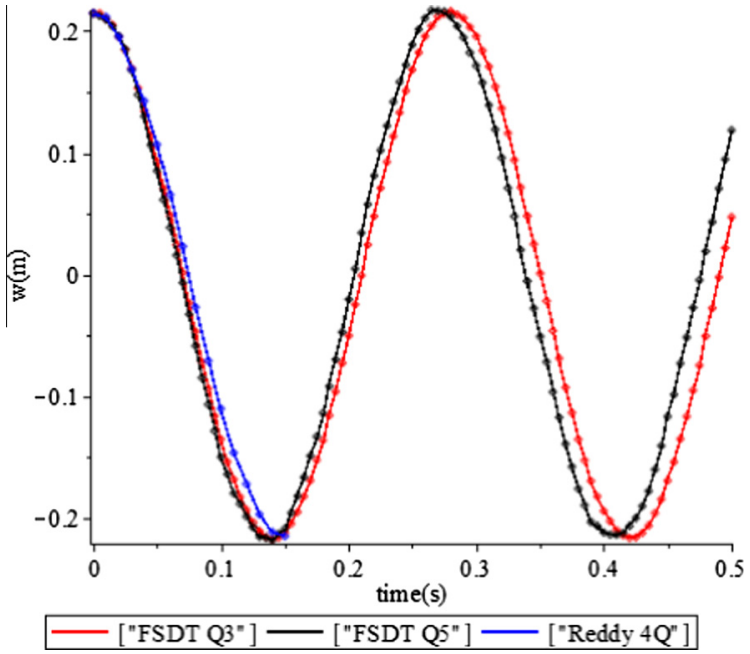

Fig. 4. Dynamic response of thin beam.

Table 4

Strip maximum deflections and fundamental frequencies.

\begin{tabular}{|c|c|c|c|c|c|c|}
\hline \multirow[t]{2}{*}{$p$} & \multicolumn{3}{|c|}{ Maximum deflection (m) } & \multicolumn{3}{|c|}{ Fundamental frequency $(\mathrm{Hz})$} \\
\hline & $\mathrm{SiC}$ & ZrO2 & $\mathrm{Al} 2 \mathrm{O} 3$ & $\mathrm{SiC}$ & ZrO2 & $\mathrm{Al} 2 \mathrm{O} 3$ \\
\hline 0 & $1.549 \mathrm{E}-6$ & & & & 1395.651 & \\
\hline 0.1 & $1.349 \mathrm{E}-6$ & $1.412 \mathrm{E}-6$ & $1.356 \mathrm{E}-6$ & 1490.403 & 1425.866 & 1477.957 \\
\hline 0.5 & $8.634 \mathrm{E}-7$ & $1.081 \mathrm{E}-6$ & $8.877 \mathrm{E}-7$ & 1845.977 & 1533.597 & 1783.151 \\
\hline 1 & $6.232 \mathrm{E}-7$ & $9.042 \mathrm{E}-7$ & $6.542 \mathrm{E}-7$ & 2159.328 & 1614.379 & 2044.326 \\
\hline 2 & $4.646 \mathrm{E}-7$ & $7.725 \mathrm{E}-7$ & $4.975 \mathrm{E}-7$ & 2485.739 & 1686.295 & 2308.658 \\
\hline 5 & $3.665 \mathrm{E}-7$ & $6.800 \mathrm{E}-7$ & $3.988 \mathrm{E}-7$ & 2782.475 & 1739.573 & 2540.992 \\
\hline 10 & $3.366 \mathrm{E}-7$ & $6.492 \mathrm{E}-7$ & $3.683 \mathrm{E}-7$ & 2895.870 & 1755.284 & 2626.810 \\
\hline
\end{tabular}

confirm that functionally graded layers incorporating silicon carbide $(\mathrm{SiC})$ provide a stiffer structure. The use of zirconia ( $\mathrm{ZrO} 2$ ) yields the less stiff configuration and thus we can find on these sandwiches the maximum deflections and the lower fundamental frequencies for any value of $p$.

If we consider not only the influence of the exponent value but additionally the $L / h$ ratio, then it is possible to obtain curves such as the two presented in Fig. 5, which illustrates two different material gradations.

Resulting from these curves, one can also conclude that the influence of the exponent value is greater and with a greater extent when we have a thicker beam, in comparison to the beam possessing a higher $L / h$ ratio. To further analyse the dynamic behavior of

Table 2

Strip maximum deflections for FSDT models.

\begin{tabular}{|c|c|c|c|c|c|c|c|}
\hline \multirow[t]{2}{*}{$L / h$} & \multirow[t]{2}{*}{$k$} & \multicolumn{6}{|c|}{ Maximum deflections (m) } \\
\hline & & Analytical [21] & FSDT L3 & FSDT L5 & FSDT Q3 & FSDT Q5 & 4CFSDT6 \\
\hline \multirow[t]{2}{*}{5} & 1 & $3.124 \mathrm{E}-8$ & $3.323 \mathrm{E}-8$ & $3.344 \mathrm{E}-8$ & $3.344 \mathrm{E}-8$ & $3.344 \mathrm{E}-8$ & \\
\hline & $5 / 6$ & $3.420 \mathrm{E}-8$ & $3.642 \mathrm{E}-8$ & $3.663 \mathrm{E}-8$ & $3.664 \mathrm{E}-8$ & $3.664 \mathrm{E}-8$ & $3.232 \mathrm{E}-8$ \\
\hline \multirow[t]{2}{*}{10} & 1 & $3.227 \mathrm{E}-7$ & $3.401 \mathrm{E}-7$ & $3.433 \mathrm{E}-7$ & $3.435 \mathrm{E}-7$ & $3.435 \mathrm{E}-7$ & \\
\hline & $5 / 6$ & $3.345 \mathrm{E}-7$ & $3.529 \mathrm{E}-7$ & $3.561 \mathrm{E}-7$ & $3.563 \mathrm{E}-7$ & $3.563 \mathrm{E}-7$ & $3.290 \mathrm{E}-7$ \\
\hline
\end{tabular}




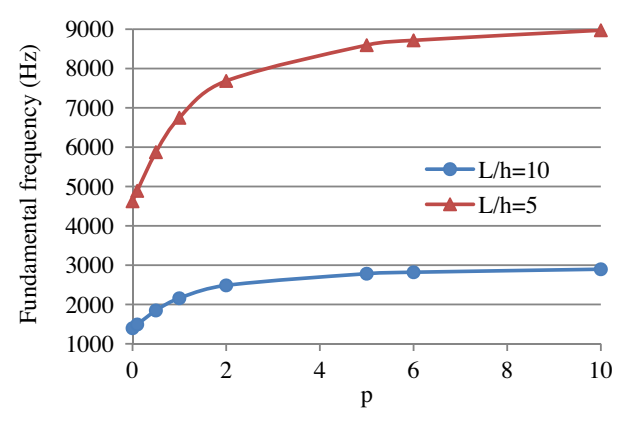

Fig. 5. Influence of $p$ and $L / h$ on fundamental frequencies.

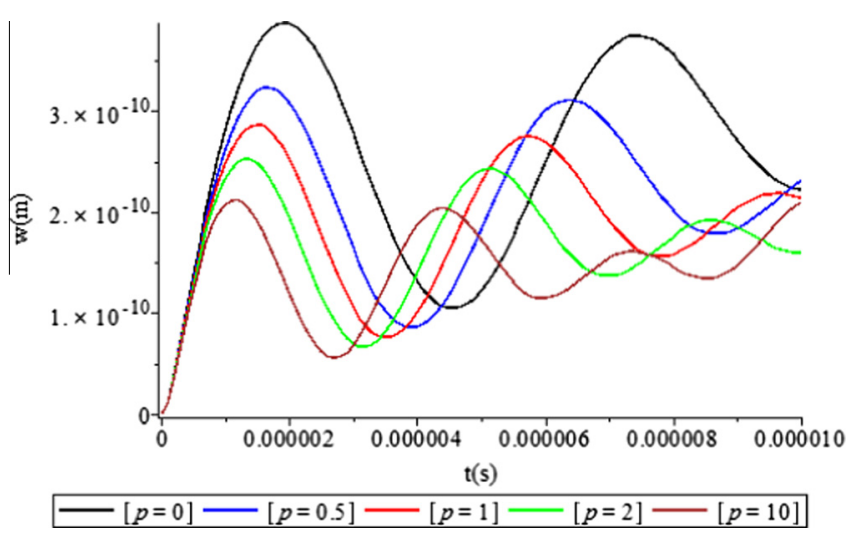

Fig. 6. Effect of power law exponent p, on transient dynamic deflection.

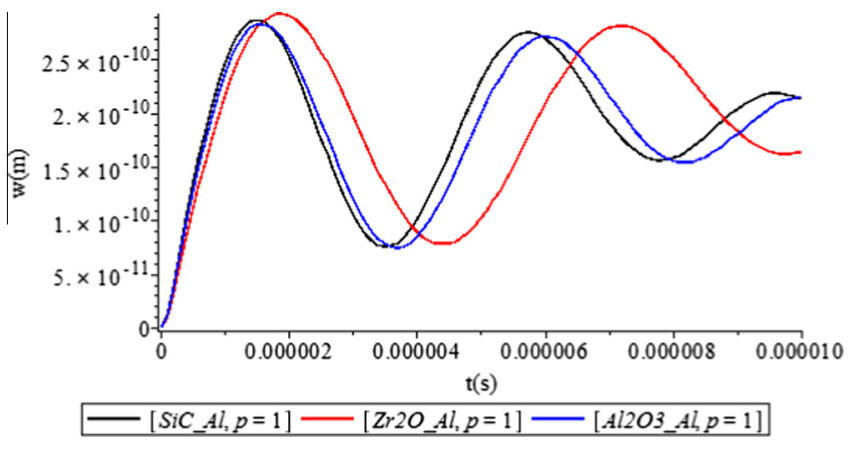

Fig. 7. Effect of material properties on transient dynamic deflection.
Table 5

Influence of the shear deformation theory on fundamental frequencies $(\mathrm{Hz})$.

\begin{tabular}{|c|c|c|c|c|c|c|}
\hline \multirow[t]{2}{*}{$p$} & \multicolumn{3}{|l|}{ FSDT Q5 } & \multicolumn{3}{|l|}{ HSDT Q5 } \\
\hline & $\mathrm{CC}$ & SS & $\mathrm{CF}$ & $\mathrm{CC}$ & SS & $\mathrm{CF}$ \\
\hline 0 & 4623.684 & 2478.655 & 918.000 & 4409.973 & 2269.271 & 839.005 \\
\hline 0.1 & 4805.436 & 2636.684 & 981.104 & 4656.405 & 2433.325 & 902.509 \\
\hline 0.5 & 5385.410 & 3201.447 & 1212.956 & 5460.423 & 3013.173 & 1131.484 \\
\hline 1 & 5893.068 & 3681.680 & 1413.176 & 6041.777 & 3480.733 & 1321.846 \\
\hline 2 & 6324.381 & 4137.350 & 1609.740 & 6572.092 & 3929.963 & 1508.963 \\
\hline 5 & 6949.896 & 4596.301 & 1794.479 & 7097.935 & 4324.676 & 1671.579 \\
\hline 10 & 7265.147 & 4786.708 & 1866.582 & 7387.231 & 4489.229 & 1734.579 \\
\hline
\end{tabular}

Table 6

Fundamental frequencies $(\mathrm{Hz})$ for cubic higher order models.

\begin{tabular}{|c|c|c|c|c|c|c|}
\hline \multirow[t]{2}{*}{$p$} & \multicolumn{3}{|l|}{ HSDT C4 } & \multicolumn{3}{|l|}{ HSDT C6 } \\
\hline & $\mathrm{CC}$ & SS & $\mathrm{CF}$ & $\mathrm{CC}$ & SS & $\mathrm{CF}$ \\
\hline 0 & 4410.054 & 2269.259 & 839.045 & 4409.156 & 2269.259 & 838.898 \\
\hline 0.1 & 4656.434 & 2433.312 & 902.547 & 4655.626 & 2433.312 & 902.403 \\
\hline 0.5 & 5460.335 & 3013.160 & 1131.529 & 5459.576 & 3013.160 & 1131.355 \\
\hline 1 & 6041.603 & 3480.719 & 1321.911 & 6040.702 & 3480.719 & 1321.678 \\
\hline 2 & 6571.812 & 3929.848 & 1509.060 & 6570.689 & 3929.848 & 1508.736 \\
\hline 5 & 7097.567 & 4324.659 & 1671.716 & 7096.272 & 4324.659 & 1671.287 \\
\hline 10 & 7386.872 & 4489.211 & 1734.734 & 7385.522 & 4489.211 & 1734.263 \\
\hline 100 & 7777.585 & 4661.292 & 1794.020 & 7776.157 & 4661.292 & 1793.521 \\
\hline
\end{tabular}

these strips, a time step of $10^{-7} \mathrm{~s}$ was used. This value is also considered in next cases. For a SiC-Al clamped-clamped strip submitted to a $10^{3} \mathrm{~N}$ step load in the first time period, and studied with the FSDT Q5 model, the answer obtained during 200 time steps is represented in Fig. 5.

In this case, we have considered $\alpha_{B}=0$, thus Newmark method was used in practice. Again the value of the exponent in the material distribution law reveals to have a significant influence. The situation that corresponds to a full-metal strip $(p=0)$ is denoted by higher transverse displacements along all the time considered. As the exponent value increases this displacement becomes smaller and the period also gets shorter.

The effect of considering different materials is also reflected on the transient dynamic answer, as can be seen in Fig. 6, where different sandwich strips were considered.

In any of the cases shown in this figure, an exponent value $p=1$ was used. Confirming the results previously obtained concerning

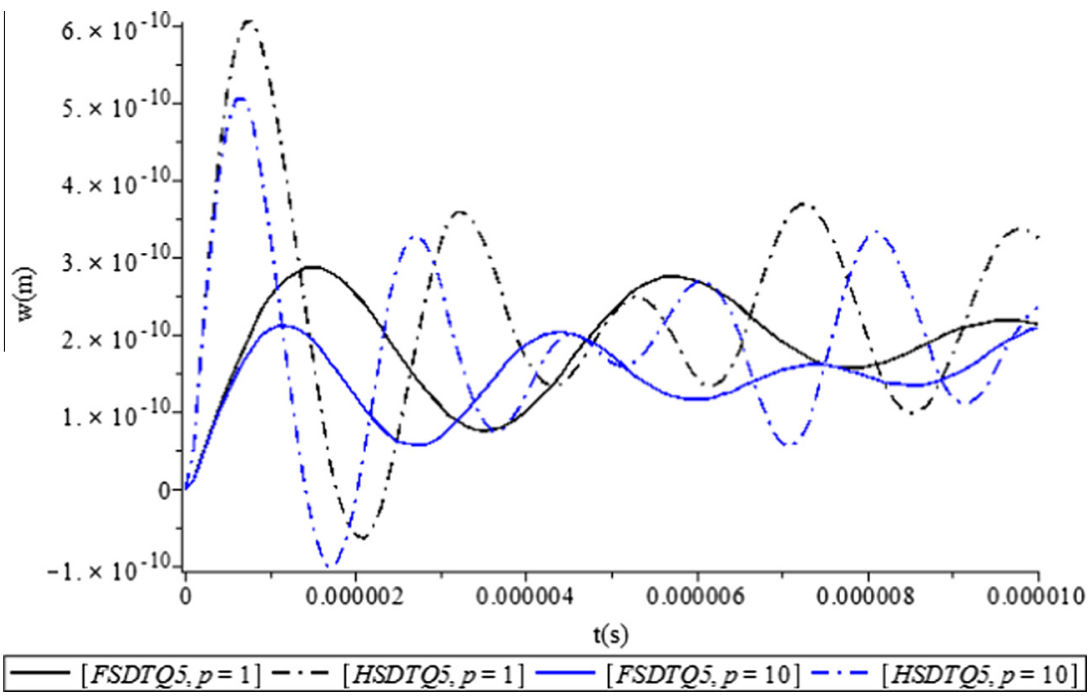

Fig. 8. Influence of shear deformation theory on dynamic response. 


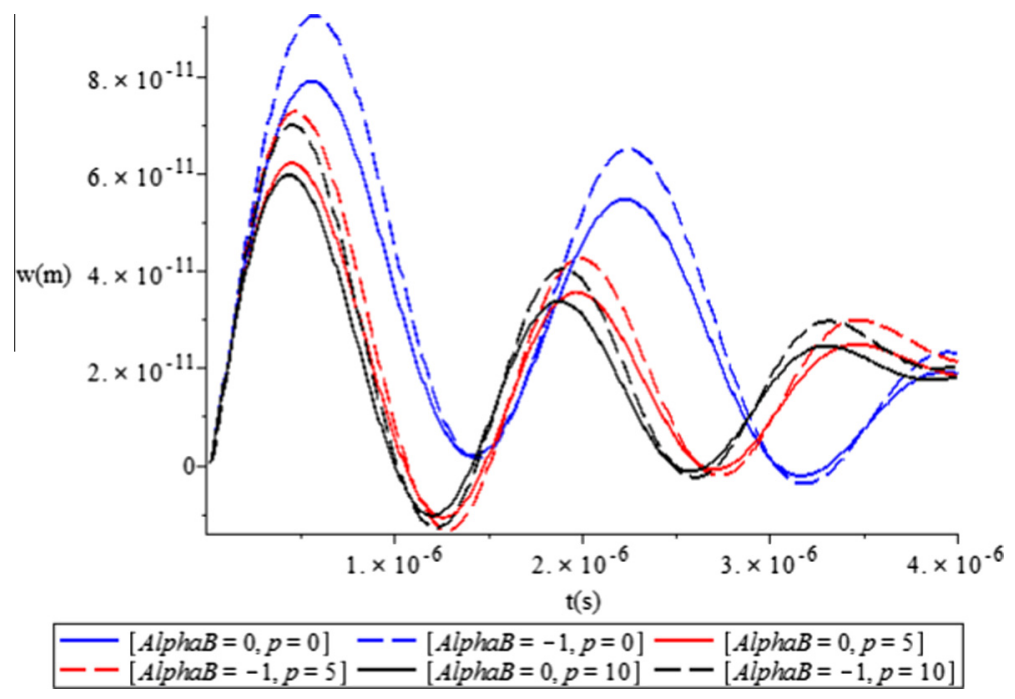

Fig. 9. Comparison between Newmark and Bossak-Newmark results $\alpha_{B}=0$.

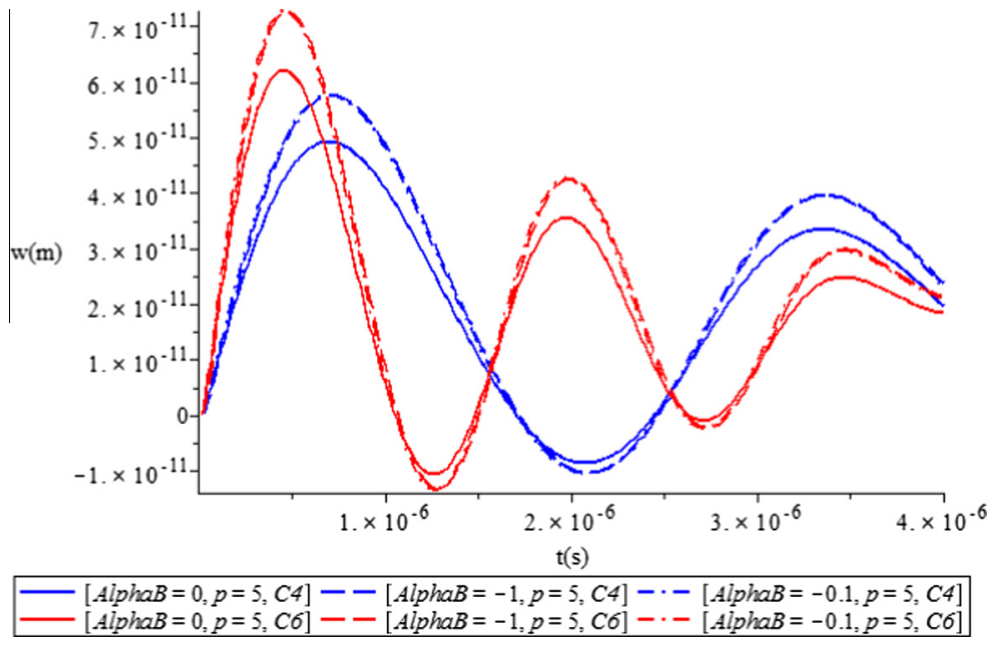

Fig. 10. Time history of higher order models.

to the static and free vibration analyses, it is clear on this figure the less stiff character of the $\mathrm{ZrO} 2-\mathrm{Al}$ sandwich structure and the stiffer structures obtained using alumina and silicon carbide (see Fig. 7).

6.5. Case 5: influence of shear deformation theories on the dynamic response

To enable a study on the influence of the shear deformation theory, without the cross-effect of using different shape functions, which is sometimes the case, we have considered FSDT Q5 and HSDT Q5, which are based on the same shape functions within the interpolation domain. In this study an $L / h$ ratio of 5 was adopted, and the silicon carbide sandwich was selected.

From this table it is possible to say that the use of first order shear deformation theory overpredicts the frequencies, when compared to the HSDT previsions. This is clearly seen in the results obtained for different boundary conditions and confirms the usual trend when using other approaches.

The transient dynamic response due to a similar step load at the initial period of time can be observed in Fig. 8 for two different values of $p$.

From the curves obtained, it is again visible the flattening effect of increasing the value of the $p$ exponent. Also the HSDT provides
Table 7

Computational time ratios among implemented models.

\begin{tabular}{|c|c|c|c|c|c|c|c|c|}
\hline \multicolumn{9}{|c|}{ Computational time ratios } \\
\hline \multirow[t]{2}{*}{ Ref. model } & \multicolumn{5}{|c|}{ FSDT } & \multicolumn{3}{|l|}{ HSDT } \\
\hline & L3 & L5 & Q3 & Q5 & Q3 & Q5 & $\mathrm{C} 4$ & C6 \\
\hline L3 & 1 & 6.67 & 1.02 & 7.27 & 3.27 & 32.91 & 13.06 & 68.09 \\
\hline Q3 & - & - & - & - & 1 & 10.06 & 3.99 & 20.81 \\
\hline
\end{tabular}

visibly higher transverse deflections when compared to the FSDT model, for this thick sandwich beam.

Considering the use of cubic polynomial basis in the context of the higher order shear deformation theory, it is also relevant to deep the understanding about the results obtained if the number of shape function in the interpolation domain is greater or not, and the differences between them. To this purpose, Table 6 presents a comparison between HSDT C4 and HSDT C6 for different boundary conditions and values of the $p$ exponent.

As can be observed in this table there exist some relative deviations that go from $0.014 \%$ to $0.028 \%$ which can be understood as negligible for this situation, and the option for a specific element in this case the HSDT C4 could be reinforced by its less expensive characteristics. A similar reasoning can be extended to the HSDT Q5, in Table 5. 
6.6. Case 6: influence of alpha value on the transient dynamic response

To enable the artificial damping of the higher modes effects it is necessary, to consider a non-zero value for $\alpha_{B}$, that is inferior or equal to $1 / 2$ to guarantee that the unconditional stability of the method is not violated. In the present case, where a simply supported $\mathrm{SiC}-\mathrm{Al}$ sandwich was analyzed, different combinations of $p$ exponent and $\alpha_{B}$ were studied. In Fig. 9, we can observe the curves corresponding to three FGM beams, with respectively $p=0$ (full metal), $p=5$ and $p=10$. For these cases, modeled with HSDT C6, we compare Newmark $\left(\alpha_{B}=0\right)$ and Bossak-Newmark solutions (in this case $\alpha_{B}=-1$ ).

For each beam considered, we can observe deviations between the maximum displacements within the time interval depending on the method applied. In fact if Bossak-Newmark method is used the corresponding maximum deflections are greater than the ones obtained using Newmark method. This is an expected pattern, taking into account the methods framework.

In Fig. 10 we can observe for the cubic HSDT kriging models, the deflections time history for different values of $\alpha_{B}$.

From these curves, we can conclude that as already mentioned, the $\mathrm{C} 4$ model has a more conservative behavior. Additionally and concerning to the influence of the $\alpha_{B}$ parameter, it is possible to conclude that the deviations between the solutions obtained when we use the values -1 or -0.1 can be considered to be not significant. Again it is visible a similar deviation pattern when we compare the Newmark solution with the Bossak-Newmark solution.

As a final remark concerning to the computational cost, it is possible to say that as expected, the higher order models are generically more expensive. To illustrate this, and taking as reference the cheaper element in each shear deformation theory (FSDT L3 and HSDT Q3 respectively) we present in Table 7 the corresponding computational time ratios.

Concerning to a specific shear deformation theory, as the number of interpolation functions used within the element domain, increase, the computational time also becomes higher. Additionally, in the case of the first order models, it can be observed the influence on the degree of the polynomial basis used.

\section{Conclusions}

The authors studied the dynamic behavior of a beam-type sandwich structure submitted to different material's graded distributions and boundary conditions. To this purpose, a package of models was developed based on first shear deformation theory and on Lo et al. higher order shear deformation theory. These models were implemented by using kriging functions based on different polynomial basis for each theory. The development of the shape functions is based on the Gaussian correlations that are established using Euclidean distances among the nodal points considered, and do not oblige these points to be equidistant, thus enabling a more flexible implementation. Also, the possibility to use these functions within an existing finite element code is a straightforward task which is an important issue considering the possibility to adequate pre-existing codes.

The average properties of the sandwich were determined using Mori-Tanaka or Voigt homogenization schemes. The dynamic behavior characterization of the sandwich functionally graded beams was carried out using Bossak-Newmark method and also by using Newmark method. The results obtained from these two methods are in accordance to the expected, where higher values of displacements are achieved when using Bossak-Newmark Globally from the tests carried out it is possible to say that the kriging models implemented provide an overall good performance, being a promising approach.

\section{Acknowledgements}

The authors wish to acknowledge the financial support given by FCT through Projects PEst-OE/EME/LA0022/2013, PTDC/ATP-AQI/ 5355/2012 and PTDC/EME/120830/2010.

\section{References}

[1] Karbowski K. Interpolation reverse engineering system. Adv Manuf Sci Technol 2008;32(4):19-30.

[2] Martin JD, Simpson TW. On the use of kriging models to approximate deterministic computer models. In: DETC2004/DAC-57300 ASME 2004 international design engineering technical conferences and computers and information in engineering conference, Salt Lake City, Utah USA, September 28-October 2, 2004.

[3] Kaimaz I. Application of kriging method to structural reliability problems. Struct Safety 2005;27(2):133-51.

[4] Laslett GM. Kriging and splines: an empirical comparison of their predicting performance in some applications. J Am Stat Assoc 1994;89(426):391-400.

[5] Loja MAR, Barbosa JI, Mota Soares CM. Optimal design of piezolaminated structures using B-spline strip finite element models and genetic algorithms. Int J Comput Eng Sci Mech 2010;11:185-95.

[6] Yang H, Lo SH, Sze KY, Leung AYT. Coupled static and dynamic buckling of thin walled beam by spline finite element. Thin Walled Struct 2012;60:118-26.

[7] Plengkhom K, Kanok-Nukulchai W. An enhancement of finite element method with moving kriging shape functions. Int J Comput Methods World Sci $2005 ; 2(4): 451-75$

[8] Bui TQ, Nguyen MN. A moving kriging interpolation-based meshfree method for free vibration analysis of Kirchhoff plates. Comput Struct 2011;89:380-94.

[9] Bui TQ, Nguyen MN, Zhang C. A moving kriging interpolation-based elementfree Galerkin method for structural dynamic analysis. Comput Methods App. Mech Eng 2011:200:1354-66.

[10] Reddy JN. Dynamic (transient) analysis of layered anisotropic compositematerial plates. Int J Numer Methods Eng 1983;19(2):237-55.

[11] Kant T, Ravichandran RV, Pandya BN, Mallikarjuna BN. Finite element transient dynamic analysis of isotropic and fibre reinforced composite plates using a higher-order theory. Compos Struct 1988;9(4):319-42.

[12] Nayak AK, Shenoi RA, Moy SSJ. Transient response of composite sandwich plates. Compos Struct 2004;64:249-67.

[13] Neves AMA, Ferreira AJM, Carrera E, Cinefra M, Roque CMC, Jorge RMN, et al. Free vibration analysis of functionally graded shells by a higher-order shear deformation theory and radial basis functions collocation, accounting for through-the-thickness deformations. Eur J Mech A/Solids 2013;37:24-34.

[14] Roque CMC, Ferreira AJM, Neves AMA, Fasshauer GE, Soares CMM, Jorge RN. Dynamic analysis of functionally graded plates and shells by radial basis functions. Mech Adv Mater Struct 2010;17:636-52.

[15] Newmark NM. A method of computation for structural dynamics. J Eng Mech Div Proc ASCE 1959:67-94.

[16] Mori T, Tanaka K. Average stress in matrix and average elastic energy of materials with misfitting inclusions. Acta Metall 1973;21(5):571-4.

[17] Reddy JN. In: An introduction to the finite element method. McGraw-Hill Education; 2005. ISBN 0072466855, 9780072466850.

[18] Lo KH, Christensen RM, Wu EM. A high-order theory of plate deformation-Part II: laminated plates. J Appl Mech 1977;44:669-76.

[19] Wood WL, Bossak M, Zienkiewicz OC. An alpha modification of Newmark's method. Int J Numer Methods Eng 1981;15:1562-6.

[20] Krige DG. A statistical approach to some basic mine valuation problems on the witwatersrand. J Chem Metall Min Soc South Africa 1951;52(6):119-39.

[21] Nguyen T-K, Sab K, Bonnet G. First-order shear deformation plate models for functionally graded materials. Compos Struct 2008;83:25-36. 